\title{
CALCULATION OF FLIGHT DECK INTERVAL MANAGEMENT ASSIGNED SPACING GOALS SUBJECT TO MULTIPLE SCHEDULING CONSTRAINTS
}

\author{
John E. Robinson III, NASA Ames Research Center, Moffett Field, California
}

\section{Abstract}

The Federal Aviation Administration's Next Generation Air Transportation System will combine advanced air traffic management technologies, performance-based procedures, and state-of-the-art avionics to maintain efficient operations throughout the entire arrival phase of flight. Flight deck Interval Management (FIM) operations are expected to use sophisticated airborne spacing capabilities to meet precise in-trail spacing from top-of-descent to touchdown. Recent human-in-the-loop simulations by the National Aeronautics and Space Administration have found that selection of the assigned spacing goal using the runway schedule can lead to premature interruptions of the FIM operation during periods of high traffic demand. This study compares three methods for calculating the assigned spacing goal for a FIM operation that is also subject to time-based metering constraints. The particular paradigms investigated include: one based upon the desired runway spacing interval, one based upon the desired meter fix spacing interval, and a composite method that combines both intervals. These three paradigms are evaluated for the primary arrival procedures to Phoenix Sky Harbor International Airport using the entire set of Rapid Update Cycle wind forecasts from 2011. For typical meter fix and runway spacing intervals, the runway- and meter fix-based paradigms exhibit moderate FIM interruption rates due to their inability to consider multiple metering constraints. The addition of larger separation buffers decreases the FIM interruption rate but also significantly reduces the achievable runway throughput. The composite paradigm causes no FIM interruptions, and maintains higher runway throughput more often than the other paradigms. A key implication of the results with respect to time-based metering is that FIM operations using a single assigned spacing goal will not allow reduction of the arrival schedule's excess spacing buffer. Alternative solutions for conducting the FIM operation in a manner more compatible with the arrival schedule are discussed in detail.

\section{Introduction}

The Federal Aviation Administration (FAA) is developing the Next Generation Air Transportation System (NextGen) which envisions advanced air traffic management technologies and procedures to accommodate safely, efficiently, and reliably the forecasted increase in traffic demand [1]. As a result of high fuel costs and a desire to leverage existing advanced aircraft equipage, a primary focus of NextGen is the use of fuel-efficient performancebased navigation $(\mathrm{PBN})$ procedures in the already dense terminal area, such as Area Navigation (RNAV) and Required Navigational Performance (RNP) Optimized Profile Descents (OPDs). In order to achieve consistent use of these advanced procedures during periods of traffic congestion, timebased scheduling will be combined with advanced ground-based and airborne spacing technologies. Time-based arrival scheduling will progressively meter the traffic flows in order to smoothly merge aircraft arriving from different directions and to avoid downstream congestion that would otherwise prevent aircraft from flying the efficient flight paths. Orderly traffic flows enable aircraft to maintain the fuelefficient PBN procedures by sustaining the use of speed adjustments to control aircraft along their routes and reducing the need to use vectoring to absorb additional delay due to excess demand or to avoid separation violations. In NextGen, air traffic controllers will use ground-based scheduling and spacing tools to accurately meet an efficient arrival schedule during periods of low, medium, and high traffic demand. Meanwhile, the best-equipped aircraft will use onboard capabilities to achieve and maintain the desired inter-arrival spacing without speed instructions from the air traffic controller.

The National Aeronautics and Space Administration (NASA) has developed its Air Traffic Management Technology Demonstration \#1 (ATD-1) concept as part of its air traffic management research [2]. ATD-1 integrates time-based scheduling throughout the entire arrival phase of flight with ground-based Controller-Managed Spacing (CMS) 
tools and airborne Flight deck Interval Management (FIM) capabilities. The time-based scheduling capabilities extend the FAA's Time-Based Flow Management (TBFM) system to include detailed modeling and scheduling of the terminal portions of the PBN arrival procedures. The CMS tools, a product of NASA's air traffic management research in the congested terminal area, provide textual and graphical representations of the arrival schedule as well as speed advisories to meet that schedule. Use of these tools enables controllers to accurately and efficiently maintain the integrity of the time-based schedule and adhere to the PBN arrival procedures. The FIM capability is an Automatic Dependent Surveillance - Broadcast (ADS-B) airborne spacing application called Airborne Spacing for Terminal Arrival Routes (ASTAR) [3]. This application provides speed commands to the flight crew in lieu of speed instructions from the air traffic controller. These speed commands enable the flight crew to maintain the schedule's arrival sequence and desired in-trail spacing while adhering to the PBN arrival procedure. Airborne spacing has been proposed as a means to achieve additional spacing precision not expected by ground-based spacing tools [4]. The eventual outcomes of ATD-1 will be the development of mature operational prototypes for both the ground and airborne systems, a series of flight trials to demonstrate the viability of the operational concept, and the transfer of the technologies to the FAA and aviation industry stakeholders.

The remainder of the paper is organized as follows. The relationship of time-based arrival scheduling and the FIM operation is explained. A simple analytical model is used to show that normal operations will routinely encounter conditions where a single spacing goal cannot satisfy the desired separation requirements. A framework is established to estimate the number of FIM interruptions that will occur for different methods for calculating a spacing goal appropriate for a time-based metering environment subject to multiple scheduling constraints. Results are presented using the primary arrival procedures to Phoenix Sky Harbor International Airport and one year of wind conditions for its terminal area. The paper concludes with recommendations for improving the compatibility of time-based metering and FIM operations with changes to both the ATD-1 operational concept and the calculation of the spacing goal.

\section{Background}

During an aircraft's arrival phase of flight, it flies through a series of airspace fixes where scheduling constraints, called metering constraints, are applied. The current FAA arrival scheduler applies scheduling constraints at two types of airspace fixes in the terminal area - the meter fix (near transition from en route to terminal airspace) and the runway threshold. At the meter fix, en route air traffic controllers are required to maintain at least 5 NM [5]; typically, they would expect an additional separation buffer of approximately 1-3 NM to avoid separation violations during transition into terminal airspace and to account for the natural compression that occurs downstream as each aircraft slows in preparation for landing. At the runway threshold, terminal air traffic controllers must maintain $2.5 \mathrm{NM}$ or $3 \mathrm{NM}$ for most aircraft pairs (see Footnote 1) depending on the runway configuration and tower operation) [5]; typically, they would expect an additional separation buffer of approximately $0.3-0.5$ NM to avoid missed approaches, go-arounds, or excessive vectoring to prevent separation violations on final approach. The ATD-1 arrival scheduler calculates scheduled times-of-arrival for each aircraft that satisfy these constraints as well as additional constraints at intermediate meter points between the meter fix and runway threshold.

For a FIM-capable aircraft, the schedule's interarrival time (i.e., in-trail spacing) is provided to the FIM aircraft for execution of airborne spacing with a so-called Target aircraft. Figure 1 illustrates the key elements of the pairwise FIM operation used for ATD-1 arrival operations. The solid green line represents the arrival procedure associated with the two aircraft. The dashed green lines represent the other arrival routes merging to the runway. The meter fix is indicated by the small black triangle. FIM is designed to achieve the assigned in-trail spacing (known as the Assigned Spacing Goal, or ASG) between the FIM and Target aircraft before the FIM aircraft crosses a downstream fix shared with the Target aircraft (known as the Achieve-By Point, or $\mathrm{ABP})$. The ASG is the conversion of the desired intrail separation at the ABP from distance to time, and it is typically expressed in terms of seconds. Throughout this paper, the term "separation" will

\footnotetext{
1 The impact of additional wake separation requirements is considered in the subsequent Discussion section.
} 
refer to the distance between the aircraft pair and the term "spacing" will refer to the time between the aircraft pair.

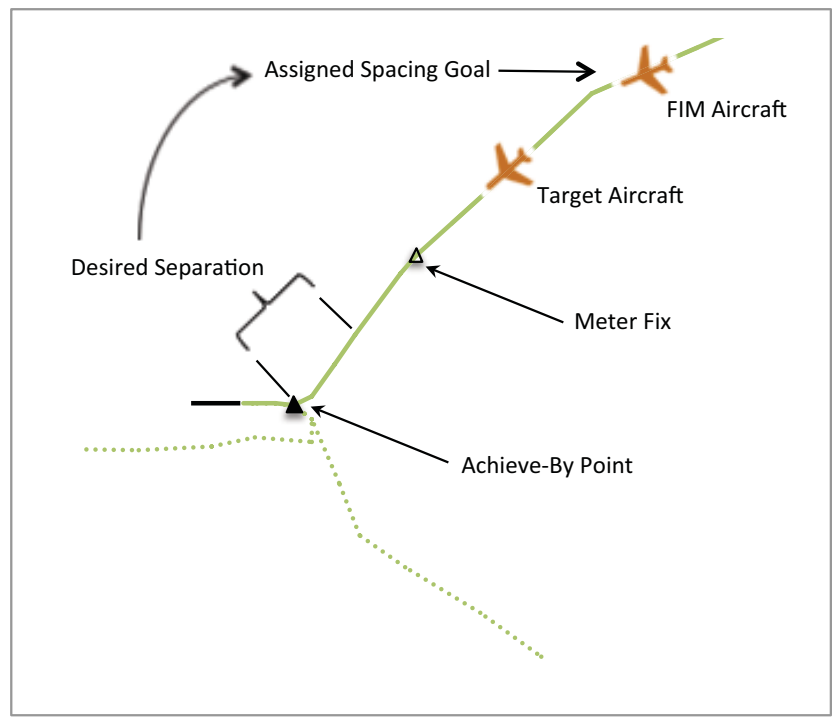

Figure 1. Key Elements of FIM Operation

In order to limit the complexity of the FIM clearance in the voice-only environment, two principal limitations are applied to the ATD-1 FIM operation. First, the ABP is prescribed to be the final approach fix (approximately $5 \mathrm{NM}$ upstream of the runway threshold). Accurate estimates of the FIM and Target aircrafts' final approach speeds and the associated compression during the remaining portion of the arrival operation are necessary to predict the downstream spacing at the runway threshold. Use of the final approach fix in lieu of the runway threshold mitigates ASTAR's lack of an accurate prediction of the Target aircraft's final approach speed (i.e., its landing speed). Instead, the ATD-1 arrival scheduler uses its detailed models of all aircraft types, including their final approach speeds, to calculate the ASG. It calculates the ASG as the difference of the FIM and Target aircrafts' scheduled times-of-arrival at the ABP. Second, a single FIM clearance, comprised of one ASG and one associated Target aircraft ID, is given by en route controllers just prior to the FIM aircraft's top-of-descent. Communication of the FIM clearance prior to descent is necessary to limit the controller and flight crew workload in the congested terminal environment. It also reduces the need for more complex changes to the terminal automation platform that are not expected to be available in the mid-term timeframe. However, the FIM and Target aircraft are not restricted to sharing a common arrival procedure since that would unnecessarily diminish the number of FIM opportunities.

The ASTAR algorithm uses a proportional control law to begin achieving the ASG immediately after initiation of the FIM operation [3]. The calculation of the speed changes necessary to meet the ASG by the ABP does not consider intermediate meter points or aircraft other than the Target aircraft. ASTAR also does not model how the required separation mandated by the current air traffic control procedures varies between en route airspace, terminal airspace, and the final approach course. Instead, the expectation is that the arrival schedule incorporates these separation constraints in a manner that allows the FIM aircraft to achieve and maintain the ASG at any time prior to the $\mathrm{ABP}$.

\section{Objective}

During recent ATD-1 human-in-the-loop simulations, air traffic controllers have occasionally interrupted or terminated the FIM operation due to unexpected compression of the FIM aircraft with its preceding or following in-trail aircraft near the meter fix. These events were observed when the FIM and Target aircraft shared the same arrival procedure as well as when they flew different arrival procedures. A natural question generated by these observations is "Can the separation at the meter fix corresponding to the ASG at the downstream ABP be less than the desired meter fix separation?" In these circumstances, the meter fix separation is a more severe scheduling constraint than the runway threshold separation.

The objective of this paper is to determine how frequently the spacing associated with the desired meter fix separation is larger than the spacing associated with the desired runway threshold separation. In these situations, the single ASG corresponding to the arrival schedule's inter-arrival time at the runway threshold will be insufficient to maintain separation at the meter fix, thus forcing the air traffic controller to interrupt or terminate the FIM operation. While the performance of ASTAR initially motivated this investigation, the issue of using a single ASG to manage spacing of traffic flows subject to multiple scheduling constraints affects any trajectory-based FIM algorithm. In order to characterize the frequency of such conditions, an analysis is performed to compare the meter fix separation (i.e., distance in-trail) associated with the 
prescribed ASG (i.e., time in-trail) to the desired meter fix separation for three ASG calculation paradigms across a large population of wind conditions. The results of this systematic analysis are used to recommend changes to the ATD-1 operational concept and arrival scheduling methods.

\section{Motivation}

Figure 2 shows the results of a simple analytical model of this potential condition. It demonstrates that the meter fix separation can be the dominant scheduling constraint for certain combinations of headwinds at the meter fix and runway threshold. These results are generated using the assumptions specified in Table 1. The green and blue lines represent the boundary between headwind conditions that have a dominant scheduling constraint at the meter fix (above the line) and those that have a dominant time constraint at the runway threshold (below the line). The results for two typical meter fix crossing speeds are shown -250 KIAS in green and 280 KIAS in blue. Only positive meter fix headwind values are shown since the runway threshold schedule constraint dominates for all negative values (i.e., meter fix tailwinds).

The dashed line represents the $95^{\text {th }}$ percentiles of the meter fix and runway threshold headwind magnitudes predicted using the National Oceanic and Atmospheric Administration's (NOAA) Rapid Update Cycle (RUC) weather forecasts for PHX in 2011. Ninety-five percent of the predicted headwinds at the runway threshold were less than eleven knots. Similarly, ninety-five percent of the predicted headwinds at the meter fix were less than forty knots.

\section{Analysis Methodology}

An empirical model is used to compare the
Table 1. Meter Fix and Runway Assumptions

\begin{tabular}{|l|l|}
\hline Meter Fix Assumptions & \\
\hline Crossing Altitude & 13,000 feet MSL \\
\hline Crossing Speeds & 250 KIAS and 280 KIAS \\
\hline Desired In-Trail Separation & 6 NM \\
\hline
\end{tabular}

Runway Assumptions

\begin{tabular}{|l|l|}
\hline Airport Elevation & Sea Level \\
\hline Final Approach Speed & 135 KIAS \\
\hline Desired In-Trail Separation & $2.8 \mathrm{NM}$ \\
\hline
\end{tabular}

desired spatial separations at two critical meter points associated with time-based arrival scheduling to the spatial separation associated with a single ASG of a FIM operation. This comparison is performed for a broad range of wind conditions at PHX using three different algorithms to calculate the ASG. Based upon models of the FIM and Target aircraft's meter fix and final approach speeds, the desired separation at these points is converted to an equivalent desired spacing. Comparison of the ASG to these desired spacing values is used to estimate how frequently FIM operations will be interrupted because of inconsistencies between the single $\mathrm{ASG}$ and the multiple scheduling constraints. The wind conditions, arrival procedure geometries, ASG calculation paradigms, and mathematical formulation of the FIM interruption rate are described in detail. The key simplifying assumptions and caveats are also discussed.

The airspace, procedures, and operations used to conduct the analyses are focused on arrival operations to Phoenix Sky Harbor International

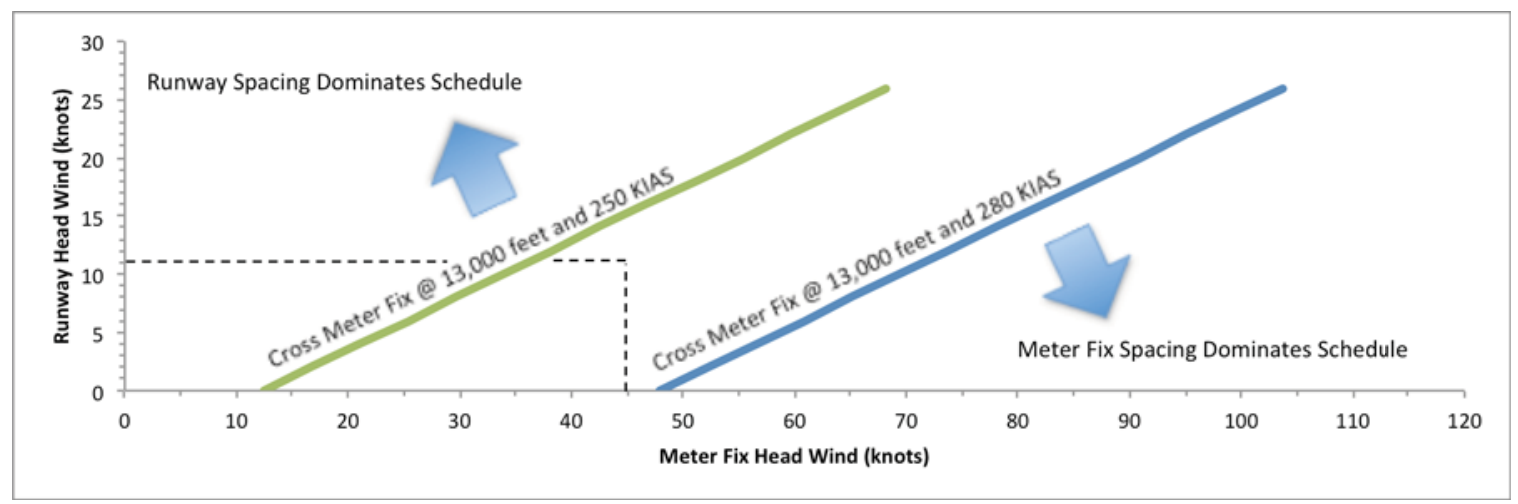

Figure 2. Comparison of Meter Fix and Runway Threshold Time Constraints 
Airport (PHX). The existing PBN arrival procedures published for PHX and the expected near-term availability of advanced air traffic control automation make it an attractive test site for research studies, lab human-in-the-loop simulations, and eventual operational demonstrations. NASA's ATD-1 Project, as well as the FAA's Terminal Sequence and Spacing (TSS) Project, have used PHX as their early development site. In addition, the wind conditions used to conduct the analyses are focused on 2011. This choice of wind conditions allows these analyses to supplement earlier ATD-1 studies that began in $2012[6][7][8]$.

\section{Wind Conditions}

A broad set of wind conditions is necessary to understand of the variations of the ASG associated with the desired separation needed for arrival operations. From 2011, a total of 8,671 individual RUC 40-km 1-hour wind forecasts are used. For the remainder of this paper, an individual RUC wind forecast will be referred to as a wind condition. These wind conditions are used to convert the desired separation to the ASG for prescribed crossing speed and altitude at the meter fix and the prescribed final approach speed and airport elevation at the runway threshold. This conversion uses the headwind component of the predicted winds in conjunction with the prescribed airspeed at the meter fix and runway threshold to calculate the inter-arrival times in a manner equivalent to the ATD-1 and FAA TBFM arrival schedulers.

The forecasted wind conditions are assumed to be representative of the actual wind conditions encountered by the arrival operation; they cover approximately $99 \%$ of 2011 . In order to ensure that the wind conditions are a reasonable reflection of landing direction, they are segregated by the winds predicted at fifty feet above the runway threshold. Subsequent results only include the subset of wind conditions having no tailwind component to the associated runway.

\section{Arrival Procedures}

As mentioned earlier, the analysis focuses on PHX arrival operations. The eight most common scenarios (4 arrival procedures $\times 2$ runways $=8$ scenarios) are analyzed with the stated wind conditions. Figure 3 shows each of these PHX arrival scenarios. The EAGUL, GEELA, KOOLY, and MAIER arrival procedures to PHX Runway 26 are shown in the top graphic; those same arrival procedures to PHX Runway 08 are shown in the bottom graphic. The PHX meter fix locations are illustrated by the small black triangles on each arrival route. The en route transition fix for each of the arrival procedures is shown by the VORTAC symbols at the start of each arrival route.
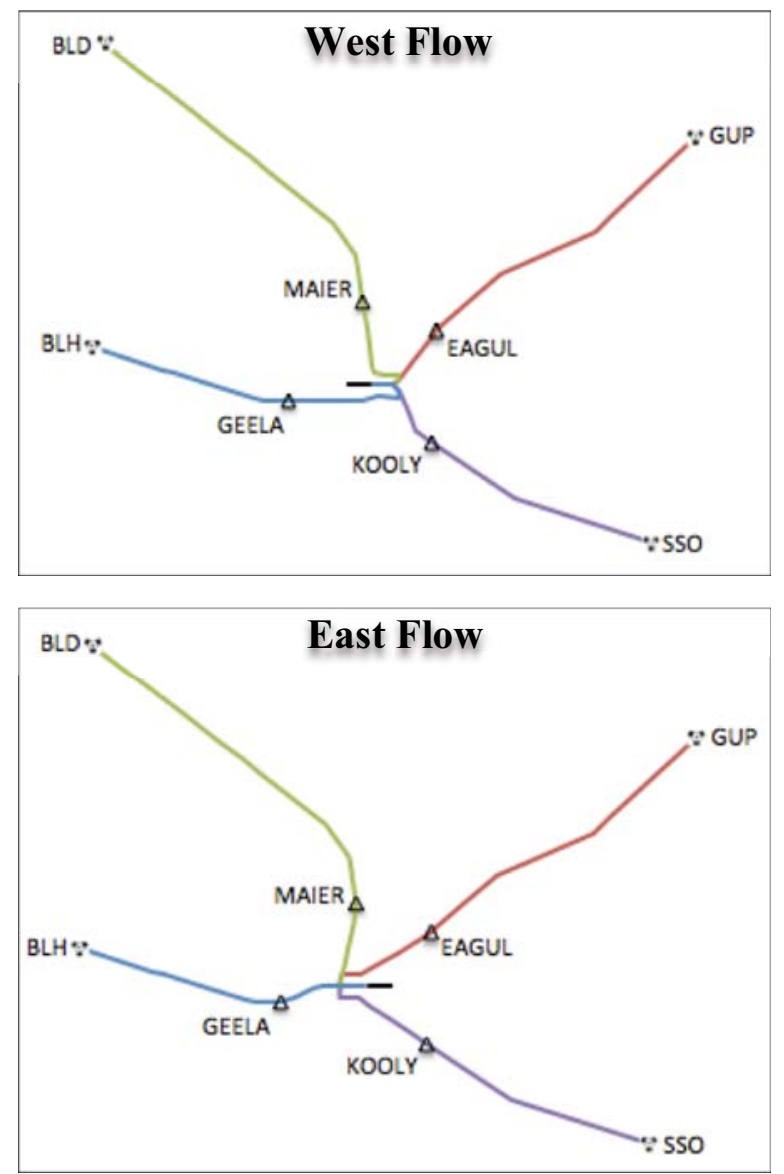

Figure 3. Arrival Procedures to PHX Runway 26 (top) and Runway 08 (bottom)

The wind conditions are segregated into East Flow and West Flow based upon the winds predicted at fifty feet above the airport surface. Wind conditions with no tailwind to PHX Runway 08 are considered East Flow, and wind conditions with no tailwind to PHX Runway 26 are considered West Flow. Subsequently, the PHX Runway 08 scenarios are analyzed using only the identified East Flow wind conditions, and PHX Runway 26 scenarios are analyzed using only the identified West Flow wind conditions. For $2011,58 \%$ of the wind conditions are 
identified as West Flow and $42 \%$ as East Flow. Incidentally, this breakdown is similar to the airport configurations reported in the FAA ASPM database (65\% West Flow and 35\% East Flow) [9].

\section{ASG Calculation Paradigms}

For each combination of arrival procedure and wind condition, three different algorithms are used to calculate the ASG. These ASG calculation paradigms are defined as follows:

\section{Runway-Based}

The ASG is calculated for the desired runway separation and no direct accommodation is made for the separation at the meter fix or intermediate terminal fixes. The FIM operation is permitted to achieve and maintain the runway-derived ASG prior to the meter fix. The analysis determines how frequently the desired meter fix spacing (i.e., the inter-arrival meter fix time associated with the desired meter fix separation) is larger than the ASG. In these circumstances, the FIM operation will need to be interrupted by controllers prior to the meter fix in order to maintain the desired meter fix separation. When not interrupted, the FIM operation would maximize runway throughput. This paradigm reflects NASA's current ATD-1 Concept of Operations [2].

\section{Meter Fix-Based}

The ASG is calculated for the desired meter fix separation and no direct accommodation is made for the runway or intermediate terminal fixes. Again, the FIM operation is permitted to achieve and maintain the meter fix-derived ASG prior to the runway. The analysis determines how frequently the desired runway spacing (i.e., the inter-arrival time associated with the desired runway separation) is larger than the ASG. In these circumstances, the FIM operation will need to be interrupted after the meter fix, but prior to the runway threshold, in order to maintain the desired runway separation. Even when uninterrupted, the FIM operation would not necessarily maximize runway throughput because the desired runway separation is not considered.

\section{Composite}

Both of the previous paradigms will encounter some FIM interruptions due to the consideration of only one of the arrival scheduling constraints. The third paradigm considers both the meter fix separation and runway separation constraints to avoid interruptions of the FIM operation. The ASG is calculated as the larger of the desired meter fix spacing and the desired runway spacing. The FIM operation will avoid meter fix separation violations by using an ASG equal to the meter fix spacing when it is larger than the runway spacing. Conversely, it will avoid runway separation violations by using an ASG equal to the runway spacing when it is larger than the meter fix spacing. In these circumstances, FIM interruptions will be minimized (by definition, there will be no schedule-related FIM interruptions) but the runway throughput will be impacted. Later, the runway performance is used to estimate how much runway efficiency and runway throughput are reduced in order to decrease the frequency of FIM interruptions.

\section{Definition of FIM Interruption Rate}

The following equations define the model used for the determination of FIM interruption rate. The static model of FIM interruption rate only considers when the ASG is inconsistent with the associated scheduling constraints. A dynamic model of FIM interruption rate, in particular one that explicitly models aircraft trajectories, their closure rates and the dynamic behavior of the FIM spacing algorithm, is deferred for future work.

The desired runway separation $\left(d_{r w y D e s}\right)$ is calculated as the sum of the required runway separation $\left(d_{r w y R e q}\right)$ and the additional runway separation buffer $\left(d_{r w y B u f}\right)$ :

$$
d_{r w y D e s}=d_{r w y R e q}+d_{r w y B u f}
$$

The desired meter fix separation $\left(d_{m f D e s}\right)$ (See footnote 2) is calculated as the sum of the required meter fix separation $\left(d_{m f R e q}\right)$ and the additional meter fix separation buffer $\left(d_{m f B u f}\right)$ :

$$
d_{m f D e s}=d_{m f R e q}+d_{m f B u f}
$$

The ASG for the runway-based paradigm $\left(A S G_{r w y}\right)$ is calculated using the desired runway separation $\left(d_{r w y D e s}\right)$, the FIM aircraft's final approach speed $\left(V_{f a}\right)$, and the predicted (i.e., RUC forecasted) headwind at fifty feet AGL at the runway threshold $\left(V_{\text {rwyHead }}\right)$ :

$$
A S G_{r w y}=d_{r w y D e s} /\left(V_{f a}-V_{r w y H e a d}\right)
$$

\footnotetext{
2 This quantity is referred to as the super stream class separation by the FAA TBFM system
} 
The effective meter fix separation associated with the $A S G_{r w y}\left(d_{m f A s g}\right)$ is calculated using the FIM aircraft's meter fix crossing speed $\left(V_{\text {cross }}\right)$, the predicted (i.e., RUC forecasted) headwind at the crossing altitude at the meter fix $\left(V_{\text {mfHead }}\right)$, and the $A S G_{r w y}$ :

$$
d_{m f A s g}=\left(V_{\text {cross }}-V_{\text {mfHead }}\right) \times A S G_{r w y}
$$

The ASG for the meter fix-based paradigm $\left(A S G_{m f}\right)$ is calculated using the desired meter fix separation $\left(d_{m f D e s}\right)$, the FIM aircraft's meter fix crossing speed $\left(V_{\text {cross }}\right)$, and the predicted headwind at the meter fix crossing $\left(V_{\text {mfHead }}\right)$ :

$$
A S G_{m f}=d_{m f D e s} /\left(V_{\text {cross }}-V_{m f H e a d}\right)
$$

The effective runway separation associated with the $A S G_{m f}\left(d_{r w y A s g}\right)$ is calculated using the FIM aircraft's final approach speed $\left(V_{f a}\right)$, the predicted headwind magnitude at fifty feet AGL at the runway threshold ( $V_{\text {rwyHead }}$ ), and the $A S G_{m f}$ :

$$
d_{r w y A s g}=\left(V_{f a}-V_{r w y H e a d}\right) \times A S G_{m f}
$$

The ASG for the composite paradigm $\left(A S G_{\text {comp }}\right)$ is calculated as the greater of the ASG for the runway-based paradigm $\left(A S G_{r w y}\right)$ and the ASG for the meter fix-based paradigm $\left(A S G_{m f}\right)$ :

$$
A S G_{\text {comp }}=\operatorname{MAX}\left(A S G_{r w y}, A S G_{m f}\right)
$$

A FIM operation is considered interrupted whenever the scheduled separation is less than the desired separation at either the runway threshold or the meter fix. Of course, this criterion is just one of many that could cause a FIM operation to be interrupted. These conditions are described as follows:

For the meter fix-based paradigm: $d_{r w y A s g}<d_{r w y D e s}$

For the runway-based paradigm: $d_{m f A s g}<d_{m f D e s}$

For the composite paradigm: never

For a particular ASG calculation paradigm, its FIM interruption rate is defined as the number of wind conditions that satisfy its FIM interruption criteria. Throughout the remainder of the paper, the FIM interruption rate will be calculated for the set of all wind conditions. Examination of the results for individual wind conditions indicates a significant seasonal variation, but further analysis of this behavior is reserved for future work.

The runway throughput ( $n_{r w y}$, landings per hour) is calculated using the ASG:

$$
n_{r w y}=A S G^{-1}
$$

where,

$$
A S G=\text { see Equation 3, } 5 \text { or } 7
$$

The runway efficiency $\left(e_{r w y}\right)$ is calculated as the ratio of the required runway inter-arrival time $\left(t_{\text {req }}\right)$ and the ASG:

$$
e_{r w y}=t_{r e q} / A S G
$$

where the required runway inter-arrival time is

$$
\begin{aligned}
& t_{\text {req }}=d_{r w y \text { Req }} /\left(V_{f a}-V_{r w y H e a d}\right) \\
& A S G=\text { see Equation } 3,5 \text { or } 7
\end{aligned}
$$

\section{Model Assumptions and Limitations}

This formulation of the FIM interruption rate, provided in Equations 8, 9 and 10, is specifically focused on those interruptions caused by using a single ASG for the entire arrival phase of flight from top-of-descent to touchdown despite there being multiple scheduling constraints. There are other possible causes for interruption of the FIM operation; these are not considered by this analysis. These simplifying assumptions and their impacts on the FIM interruption rate are discussed next.

First, the meter fix crossing and final approach speeds are modeled as constants. The meter fix crossing speed $\left(V_{\text {cross }}\right)$ is specified as 250 KIAS in order to match the typical speeds of PBN procedures, inter-facility letters of agreement, and facility standard operating procedures. The final approach speed $\left(V_{f a}\right)$ is specified as 135 KIAS in order to match the typical final approach speed of large commercial jets. As shown in Figure 2, faster meter fix crossing speeds and slower final approach speeds will increase the frequency that the runway threshold time constraint is dominant.

Second, the ASG is presumed to be satisfied precisely for both the meter fix and runway threshold. In other words, the spacing interval at the meter fix is equal to the ASG (i.e., no spacing error exists at the meter fix) and no spacing error is introduced between the meter fix and runway threshold. If the spacing errors at the meter fix are non-zero, the FIM interruption rates will be improved or degraded depending upon the specific statistical distribution of the spacing errors at that point.

Third, wind forecast errors are not modeled. Wind forecast errors affect the conversion of the 
desired separation to the ASG. The wind forecast errors are assumed to be unbiased (i.e., have a zero mean). Also, the magnitude of the time-of-arrival errors due to wind forecast errors are assumed to be less than the aircraft's speed control authority. Therefore, the presumption is that the aggregate FIM interruption rate (i.e., the average FIM interruption rate for a large number of wind forecasts) will not be affected. The wind errors of some forecasts will cause additional FIM interruptions and some will avoid interruptions predicted without consideration of the wind errors.

Fourth, the analysis of FIM operations is limited to aircraft pairs arriving along the same arrival procedure; this situation is particularly relevant for several reasons. First, the FAA's FIM Concept of Operations expects FIM operations on the same arrival procedure to be the earliest implementation of FIM procedures in the NAS [10]. This scenario avoids a main source of prediction uncertainty - the predicted winds aloft - so the FIM operations are expected to attain their highest spacing performance. It also provides the earliest control horizon, since ADS-B range is not a factor as the aircraft are naturally in relatively close proximity.

Lastly, the FIM operation is assumed to be interrupted simply when the separation associated with the ASG is less than the desired separation (e.g., Equations 8, 9, and 10). Another possible criteria is to assume that the FIM operation is not interrupted until the separation associated with the ASG is less than the required separation. This alternative criterion would reduce the FIM interruption rate. However, controllers would still realistically interrupt the FIM operation at some "comfortable" distance prior to a separation violation. This implicit FIM interruption buffer remains to be determined.

\section{Results}

The various ASG calculation paradigms are analyzed from two perspectives using the four PHX RNAV arrival procedures, both PHX landing directions and 8,671 RUC wind forecasts from 2011 (approximately 70,000 combinations). First, the FIM interruption rate is analyzed for the runway- and meter fix-based ASG calculation paradigms; it is not shown for the composite ASG calculation paradigm because the most restrictive spacing constraint is used to avoid all schedule-related FIM interruptions.
Second, the runway efficiency and runway throughput of the three paradigms is analyzed for various desired meter fix and runway separation values.

\section{Interruption Rate of Runway-Based Paradigm}

Figure 4 shows the frequency of FIM interruptions over a one-year period for different meter fix separation values and a desired runway separation of $2.8 \mathrm{NM}\left(d_{r w y R e q}=2.5 \mathrm{NM}, d_{r w y B u f}=0.3\right.$ $\mathrm{NM}$ ) using the runway-based ASG calculation paradigm. This prescribed runway separation corresponds to the value used in NASA's ATD-1 simulations as well as the FAA's TSS simulations. As expressed by Equation 9, a FIM interruption is declared when the meter fix separation associated with the runway-based ASG $\left(d_{m f A s g}\right)$ is less than the desired meter fix separation $\left(d_{m f D e s}\right)$. These FIM interruptions would occur prior to the meter fix in order to preserve the desired meter fix separation. The results for West Flow (PHX Runway 26) and East Flow (PHX Runway 08) are shown on the top and bottom plots, respectively. The solid black line represents the results for all arrival procedures across all wind conditions; the dashed blue lines represent the results for individual arrival procedures, as noted, across all wind conditions.

Currently, the FAA's time-based metering scheduler uses 6,7 , or $8 \mathrm{NM}$ for its desired meter fix separation of turbojet arrivals at most airports. For West Flow and East Flow, 73\% of all combinations of arrival procedure and wind condition are predicted to have meter fix separations less than $7 \mathrm{NM}$. NASA's ATD-1 simulations are currently using 6 $\mathrm{NM}$ for the desired meter fix separation. Approximately $5 \%$ of the cases are still predicted to have too little meter fix separation when the desired meter fix separation is reduced to $6 \mathrm{NM}$. However, no cases are predicted to have meter fix separation less than the en route minimum separation of 5 NM. Therefore, FIM operations for some cases are interrupted in order to maintain the desired meter fix separation, but none are predicted to result in losses of required separation.

The FIM interruption rate is substantially reduced when standard runway separation (3 NM) rather than reduced runway separation $(2.5 \mathrm{NM})$ is used. For these conditions (not shown), only $4 \%$ of all cases are predicted to have meter fix separations 
less than 7 NM. Moreover, only one of the cases $(0.03 \%)$ is predicted to have meter fix separations less than $6 \mathrm{NM}$ and none less than the en route minimum separation of $5 \mathrm{NM}$. However, most busy airports have the necessary infrastructure and operational procedures to allow reduced runway separation. Therefore, the throughput reduction associated with increasing the desired runway separation, in terms of runway efficiency, will be discussed later.

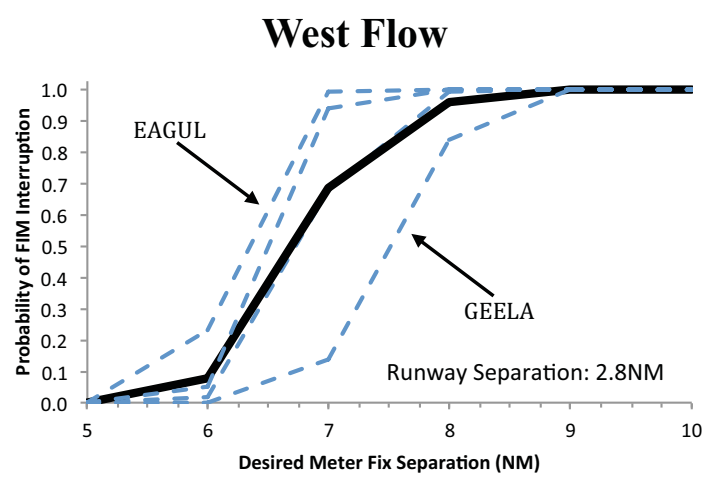

East Flow

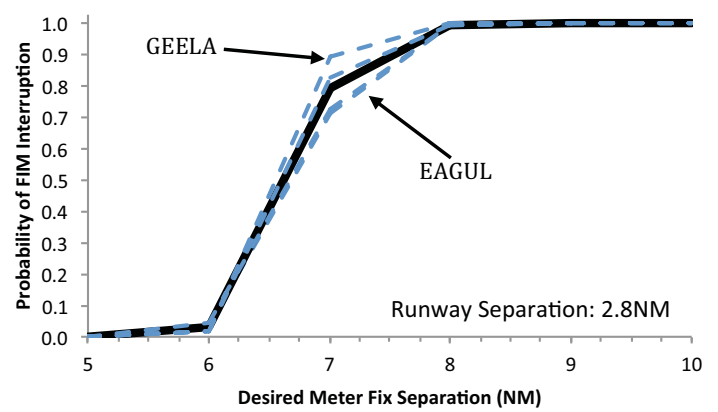

\section{Figure 4. Probability of FIM Interruption for Runway-Based Paradigm}

The results for West Flow and East Flow are presented separately in order to illustrate the differences related to the airport configuration which is related to the winds at the airport. Investigation of the wind magnitudes and directions suggests that the greater variability of the West Flow winds relative to the East Flow winds across the arrival routes causes the greater variability of the FIM interruption rate across the arrival routes. Overall, the range of wind speeds predicted at meter fix crossing is more similar across the East Flow arrival routes than the West Flow arrival routes. Thus, the results for the individual arrival routes (the dashed blue lines in Figure 4 and Figure 5) are more similar in East Flow than in West Flow. Examination of the wind conditions shows that relative to the other arrival routes in both East Flow and West Flow, the West Flow GEELA arrivals have stronger predicted tailwinds while the West Flow EAGUL arrivals have stronger predicted headwinds. Understanding the airspace-dependent impact of the environmental conditions on the FIM operation is an important factor to their uninterrupted execution.

\section{Interruption Rate of Meter Fix-Based Paradigm}

Figure 5 shows the frequency of FIM interruptions for different runway separation values and a desired meter fix separation of $6 \mathrm{NM}\left(d_{m f R e q}=5\right.$ $\mathrm{NM}, d_{m f B u f}=1 \mathrm{NM}$ ) using the meter fix-based ASG calculation paradigm. This prescribed meter fix separation corresponds to the values used in NASA's ATD-1 simulations. As expressed by Equation 8, a FIM interruption is declared when the runway separation associated with the meter fix-based ASG $\left(d_{r w y A s g}\right)$ is less than the desired runway separation $\left(d_{r w y D e s}\right)$. These FIM interruptions would occur inside the meter fix and prior to the runway in order to preserve the desired runway separation. The results are presented in the same manner as Figure 4. The results for West Flow and East Flow are shown on the top and bottom, respectively. The solid black line represents all arrival procedures across all wind conditions; the dashed blue lines represent individual arrival routes, as noted, across all wind conditions.

Mathematically, the results of the meter fixbased paradigm are the complement of the runwaybased paradigm's results. For a desired runway separation of $2.8 \mathrm{NM}$, approximately $95 \%$ of the combinations of arrival procedure and wind condition are predicted to have runway separations that are less than the desired runway separation. Unlike the runway-based paradigm, the meter fix-based paradigm also has some cases predicted to have runway separation less than the minimum runway separation of $2.5 \mathrm{NM}$. In particular, almost $98 \%$ of the GEELA West Flow arrivals do not maintain the minimum runway spacing when the ASG corresponds to a desired meter fix separation of 6 NM. This percentage would rise for aircraft pairs needing larger separation at the runway threshold.

Like the runway-based paradigm, increasing the desired meter fix separation from $6 \mathrm{NM}$ to $7 \mathrm{NM}$ 
reduces the FIM interruption rate (not shown). Only $21 \%$ of cases are still predicted to have runway separation less than a desired runway separation of 2.8 $\mathrm{NM}$ in this situation. The throughput reduction associated with increasing the desired meter fix separation, in terms of runway efficiency, will be discussed later.

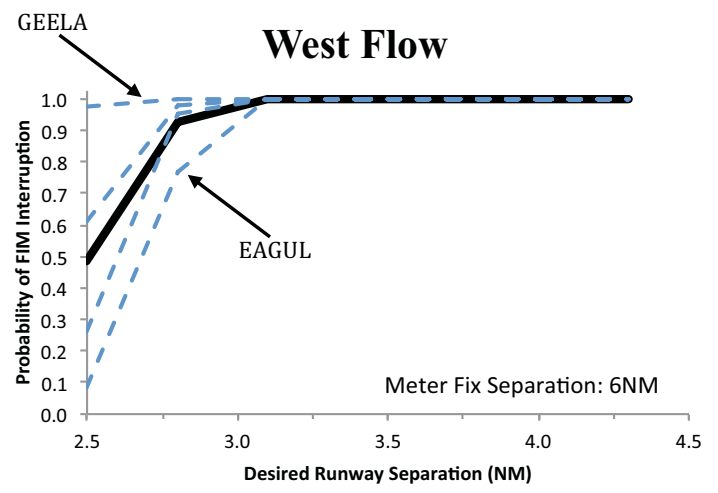

East Flow

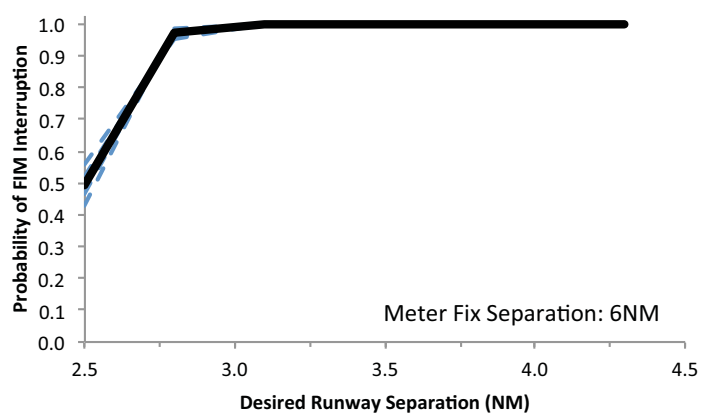

Figure 5. Probability of FIM Interruption for Meter Fix-Based Paradigm

\section{Runway Efficiency of ASG Paradigms}

Three scenarios are analyzed to understand the runway throughput impacts of the different ASG calculation paradigms. For each paradigm, the desired meter fix and runway separation values are selected to ensure that $99.7 \%$ of the combinations of arrival procedure and wind condition are uninterrupted (i.e., the FIM interruption rate is restricted to a " 3 -sigma event"). For all of these cases, the required runway separation is $2.5 \mathrm{NM}$, and the required meter fix separation is $5 \mathrm{NM}$. The runwaybased paradigm uses an ASG associated with the desired runway separations shown in Table 2; the meter fix-based paradigm uses an ASG associated with the designated meter fix separations shown in Table 3; and the composite paradigm uses an ASG corresponding to the larger of a desired meter fix separation of $6 \mathrm{NM}$ and a desired runway separation of $2.8 \mathrm{NM}$ to avoid both meter fix separations less than $6 \mathrm{NM}$ and runway separations less than 2.8 NM.

Table 2. Desired Runway Separation for the Runway-Based Paradigm $(p<0.3 \%)$

\begin{tabular}{|c|c|c|}
\cline { 2 - 3 } \multicolumn{1}{c|}{} & East Flow & West Flow \\
\hline EAGUL & $2.95 \mathrm{NM}$ & $3.01 \mathrm{NM}$ \\
\hline GEELA & $2.90 \mathrm{NM}$ & $2.67 \mathrm{NM}$ \\
\hline KOOLY & $2.91 \mathrm{NM}$ & $2.96 \mathrm{NM}$ \\
\hline MAIER & $3.01 \mathrm{NM}$ & $2.98 \mathrm{NM}$ \\
\hline
\end{tabular}

Table 3. Desired Meter Fix Separation for the Meter Fix-based Paradigm $(\mathbf{p}<0.3 \%)$

\begin{tabular}{|c|c|c|}
\cline { 2 - 3 } \multicolumn{1}{c|}{} & East Flow & West Flow \\
\hline EAGUL & $8.53 \mathrm{NM}$ & $7.18 \mathrm{NM}$ \\
\hline GEELA & $7.35 \mathrm{NM}$ & $9.02 \mathrm{NM}$ \\
\hline KOOLY & $8.16 \mathrm{NM}$ & $7.48 \mathrm{NM}$ \\
\hline MAIER & $8.19 \mathrm{NM}$ & $8.20 \mathrm{NM}$ \\
\hline
\end{tabular}

Figure 6 shows the cumulative probability of runway efficiency (see Equation 12) across all of the 2011 wind conditions for the three scenarios. The results for West Flow and East Flow are shown on the top and bottom, respectively. The solid blue line represents the runway-based paradigm for all arrival procedures, the solid green line represents the meter fix-based paradigm; and the solid orange line represents the composite paradigm. These statistical distributions exclude the combinations of arrival procedure and wind condition predicted to be interrupted (approx. 0.3\%).

There are two benchmark values for runway efficiency - the ideal runway efficiency and the schedule runway efficiency. The ideal runway efficiency has a value of unity and corresponds to a required runway separation of $2.5 \mathrm{NM}$ using no runway separation buffer $\left(d_{r w y R e q}=2.5 \mathrm{NM}\right.$ and $d_{r w y B u f}$ $=0 \mathrm{NM})$. The scheduled runway efficiency has a value of 0.89 and is simply the ratio of the required runway separation and the desired runway separation using the default runway separation buffer $\left(d_{r w y R e q}=\right.$ $2.5 \mathrm{NM}$ and $d_{r w y B u f}=0.3 \mathrm{NM}$ ). As shown in Figure 6, none of the paradigms exceed a runway efficiency of 
0.89 due to the $0.3 \mathrm{NM}$ runway separation buffer $\left(d_{r w y R e q}=2.5 \mathrm{NM}\right.$ and $\left.d_{r w y B u f}=0.3 \mathrm{NM}\right)$.

\section{West Flow}

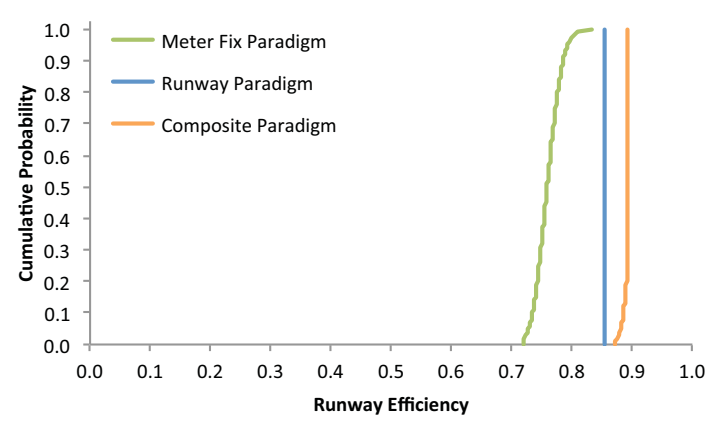

East Flow

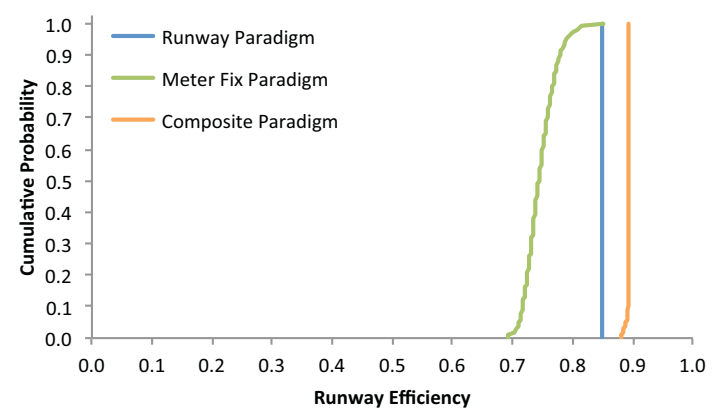

Figure 6. Probability of Runway Efficiency for Different Paradigms

By definition, the runway efficiency of the runway-based paradigm is constant for any particular desired runway separation. For the values specified in Table 2, the runway-based paradigm's mean runway efficiency across each arrival procedure is 0.86 for West Flow and 0.85 for East Flow. In other words, its mean runway efficiency is approximately $85 \%$ of the ideal runway efficiency and approximately $96 \%$ of the scheduled runway efficiency.

The runway efficiency of the meter fix-based paradigm is a well represented by a normal distribution. For the values specified in Table 3, the mean and standard deviation of the runway efficiency across each arrival procedure are $[\mu=0.76, \sigma=$ 0.0019] for West Flow and $[\mu=0.75, \sigma=0.025]$ for East Flow. Thus, the mean runway efficiency of the meter fix-based paradigm is $11-12 \%$ lower than the runway efficiency of the runway-based paradigm. Moreover, the relatively small standard deviations cause all West Flow and East Flow cases to have higher runway efficiency for the runway-based paradigm than for the meter fix-based paradigm.
By combining the statistical behavior of the runway- and meter fix-based paradigms, the composite paradigm achieves higher runway efficiency. A portion of the statistic distribution is similar to the results of the normally distributed meter fix-based paradigm and the remainder is similar to the constant runway-based paradigm. The mean runway efficiency of the composite paradigm is increased to 0.89 - an improvement of approximately $4 \%$ compared to the runway-based paradigm. Another important characteristic of the composite paradigm is that $73 \%$ of the West Flow cases and $90 \%$ of the East Flow cases have a runway efficiency equaling the scheduled runway efficiency. Thus, its statistical distribution has a median value equal to the scheduled runway efficiency.

\section{Runway Throughput of ASG Paradigms}

The analysis of runway efficiency shows distinct differences in the performance achieved by the three ASG calculation paradigms. Two other aspects of their performance - the mean runway throughput and its $95 \%$ central range - are also analyzed. The same desired meter fix and runway separation values specified in Table 2 and Table 3 are used for each paradigm.

Figure 7 shows the cumulative probability of runway throughput for the three paradigms. The results for West Flow and East Flow are shown on the top and bottom, respectively. The solid blue line represents the runway-based paradigm for all arrival procedures, the solid green line represents the meter fix-based paradigm; and the solid orange line represents the composite paradigm. Like Figure 6, these statistical distributions exclude the combinations of arrival procedure and wind condition predicted to be interrupted (approx. 0.3\%).

During West Flow and East Flow, the mean runway throughput is 45-46 aircraft per hour for the runway-based paradigm and 40-41 aircraft per hour for the meter fix-based paradigm. Like the runway efficiency results, the mean throughput of the runway-based paradigm is estimated to be $10-15 \%$ higher than the mean throughput of the meter fixbased paradigm. The composite paradigm achieves a substantially higher mean throughput of 48 aircraft per hour. This difference represents an additional $10 \%$ increase in the runway capacity that can be scheduled while avoiding FIM interruptions due to 
use of a single ASG from top-of-descent to touchdown.

\section{West Flow}

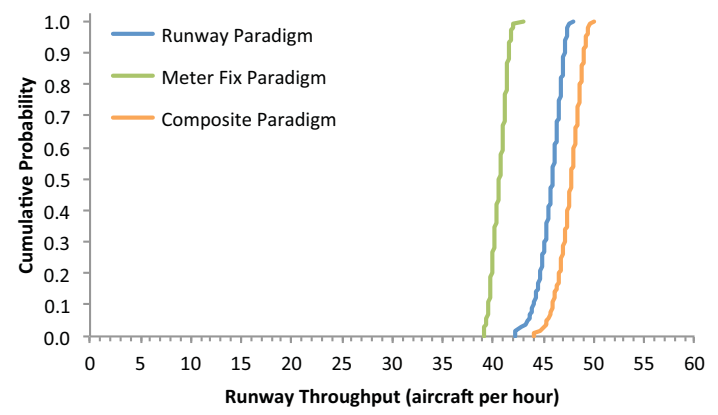

East Flow

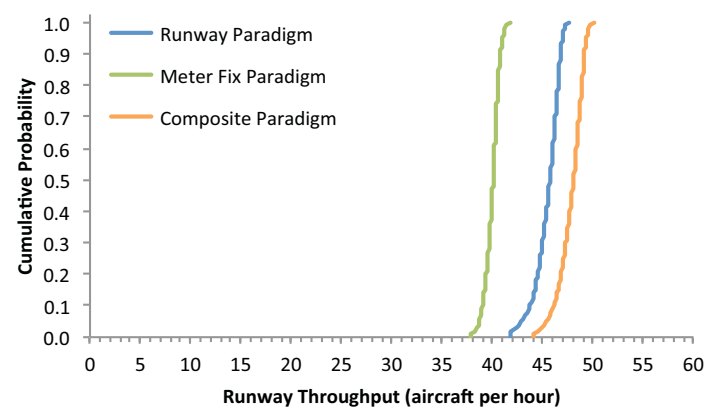

Figure 7. Probability of Runway Throughput for Different Paradigms

In addition to the mean runway throughput, the 95\% central range is used to investigate the hour-tohour capacity variations of the three paradigms. A larger value means that the effective runway capacity is more sensitive to the winds when targeting a particular FIM interruption rate. Depending upon the expected arrival demand profile for the airport, the runway would exhibit corresponding hour-to-hour variations in arrival delays. The range for the meter fix-based paradigm was smallest $-2-3$ aircraft per hour. Conversely, the range for the runway-based and composite paradigms were greatest and very similar 4-5 aircraft per hour. The smaller $95 \%$ central range and lower runway efficiency of the meter fix-based paradigm means that it is not responding to the wind conditions that would otherwise allow more aircraft to land.

The previous results can be combined to evaluate the separation buffers associated different desired FIM interruption rates. Figure 7 shows that the runway efficiency of the runway-based and meter fix-based paradigms fall short of the composite paradigm when FIM interruption rate is limited to $0.3 \%$. The same methodology is used to evaluate the runway throughput for different FIM interruption rates. Figure 8 shows the meter fix and runway throughputs that yield $31.8 \%$ (i.e., a "1-sigma event"), $4.6 \%$ (i.e., a "2-sigma event"), and $0.3 \%$ (i.e., a 3sigma event") FIM interruption rates for the meter fix- and runway-based paradigms, respectively. These results are an average of each arrival procedure's corresponding runway throughput. The results for West Flow and East Flow are shown on the top and bottom, respectively. The solid blue line represents the runway-based paradigm for all arrival procedures, the solid green line represents the meter fix-based paradigm; and the solid orange line represents the composite paradigm. The separation buffers for the composite paradigm are constant $\left(d_{r w y B u f}=0.3 \mathrm{NM}\right.$ and $\left.d_{m f B u f}=1 \mathrm{NM}\right)$ since its FIM interruption rate is not variable.
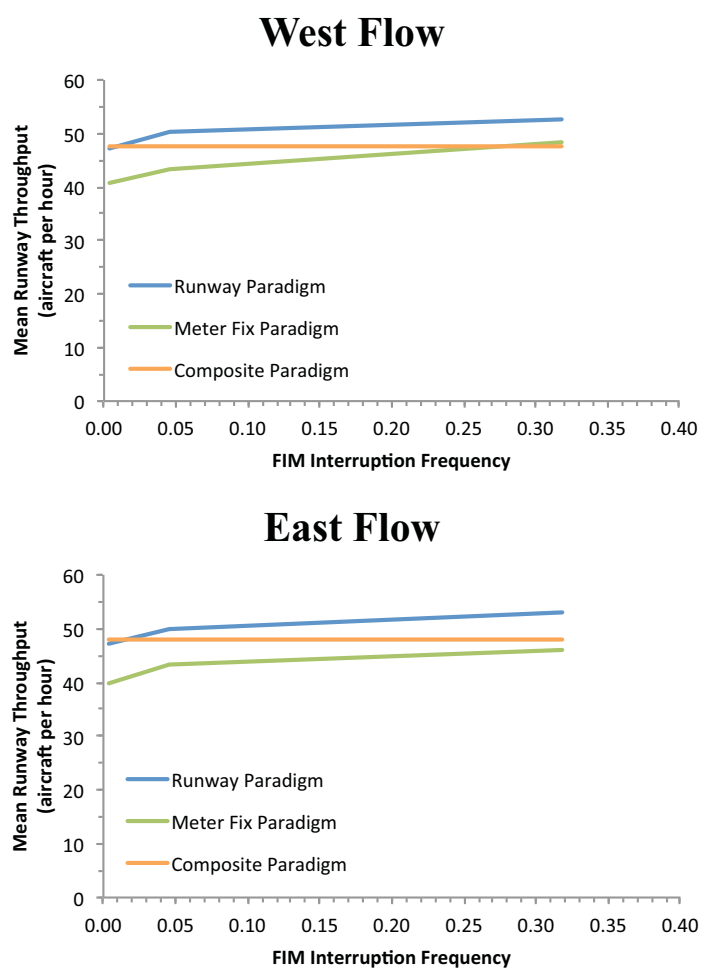

\section{Figure 8. Runway Throughput for Different FIM Interruption Rates}

There is a small improvement in mean runway throughput for the runway- and meter fix-based paradigms as the targeted FIM interruption rate is increased. However, the improvement is nearly linear for both paradigms, so increasing the FIM 
interruption rate does not substantially change their relative performance. When the FIM interruption rate is increased to $4.6 \%$, the runway-based paradigm achieves the scheduled runway throughput (represented by the composite paradigm). Moreover, when the FIM interruption rate is increased to $31.8 \%$, all three paradigms achieve the scheduled runway throughput.

\section{Discussion}

This section discusses the sensitivity of the FIM interruption rate to factors that are not modeled by this analysis. These factors include additional wake separation requirements (greater than $3 \mathrm{NM}$ ), the assumed meter fix crossing and final approach speeds, FIM pairing across different arrival streams, and scheduling constraints at intermediate terminal fixes.

\section{Effect of Additional Wake Separation}

Only the most common required runway separation distances (i.e., 2.5 NM and $3 \mathrm{NM}$ ) are analyzed in the preceding results. Other separation distances, such as those needed for wake separation, are not considered. There is less homogeneity of the required traffic separation in actual operations. However, a more varied traffic mix does not affect the results of the roughly $80 \%$ of arrival aircraft pairs associated with the modeled separation distances [11]. In general, the various paradigms will be affected differently. For example, prior ATD-1 simulations often modeled the FIM and Target aircraft as Boeing $757 \mathrm{~s}$ with a required runway separation of $4 \mathrm{NM}$ for wake separation [12]. For this pair, the FIM interruption rate of the runway-based paradigm is less because the desired runway separation is increased by $1 \mathrm{NM}$ (mathematically equivalent to an additional 1 NM runway separation buffer). As discussed in earlier, even the modest increase from reduced runway separation $(2.5 \mathrm{NM})$ to standard runway separation ( $3 \mathrm{NM}$ ) is able to ensure that the desired runway spacing is greater than the desired meter fix spacing for more than $99 \%$ of the wind conditions studied. Conversely, the FIM interruption rate of the meter fix-based paradigm will worsen for the same reason.

\section{Sensitivity to Trajectory Modeling Parameters}

The conversion of the desired separation distance to its corresponding spacing interval uses a meter fix crossing speed of 250 KIAS and final approach speed of 135 KIAS. These speeds are consistent with the commonly published arrival procedures and modeled speed profiles of large commercial jets. A reduction in either of these speeds will affect the corresponding spacing - either at the runway threshold or meter fix. Equations 3 and 5 show that use of a slower speed to calculate the ASG will affect the FIM interruption rate in the same manner as an increase of the separation buffer. For example, reducing the final approach speed from 135 KIAS to 130 KIAS is numerically equivalent to increasing the runway separation buffer from $0.3 \mathrm{NM}$ to $0.31 \mathrm{NM}(135: 130=0.3115: 0.30)$. For the runway-based paradigm (see Equations 4 and 9), a slower final approach speed will decrease the number of FIM interruptions at the meter fix, because the ASG will be greater. Conversely, for the meter fixbased paradigm (see Equations 6 and 8), a slower meter fix crossing speed will increase the ASG and thus decrease the number of FIM interruptions at the runway threshold.

\section{Extension to Additional Scheduling Constraints}

The desired meter fix and runway separation are the two principal scheduling constraints. The current FAA arrival scheduler does not directly consider the impact of fixes between the meter fix and runway threshold. However, separation at these intermediate fixes can be more time constraining under certain conditions (depending upon the aircraft's speed profile and the altitude profile of the winds encountered during descent). In other words, the spacing associated with the required separation at one of the meter points between the meter fix and runway threshold might exceed the meter fix spacing while the runway spacing does not. For example, reduced runway separation is only permitted within $10 \mathrm{NM}$ of the runway threshold for aircraft established on the final approach course [5]. Depending upon the particular aircraft speed profiles, the transition from 3 $\mathrm{NM}$ terminal separation to $2.5 \mathrm{NM}$ reduced separation will have a similar impact on the FIM interruption rate as the transition from $5 \mathrm{NM}$ en route separation to $3 \mathrm{NM}$ terminal separation at the meter fix. This situation is expected to be less frequent but should be verified by additional analysis. The analysis methodology described above can be extended to examine the FIM interruptions due to these other scheduling constraints. Moreover, the 
composite paradigm could be expanded to use the largest spacing requirement of the entire set of scheduling constraints, if necessary.

\section{Implications for Time-Based Metering}

This section discusses the implications of these results on time-based metering of FIM operations. Recommendations are provided that balance the conflicting desires to maintain high runway arrival rates (i.e., high runway efficiency) and low FIM interruption rates. The implications of these recommendations are briefly discussed in regards to schedule consistency and expected FIM benefits.

A major benefit presumed of FIM operations is a smaller runway separation buffer due to the increased inter-arrival precision between the FIM and Target aircraft that cannot be achieved by ground-based spacing tools [13]. The model of this improvement used for recent cost-benefit assessments has been a spacing buffer reduction from $0.3 \mathrm{NM}$ expected for ground-based spacing tools like TSS to $0.2 \mathrm{NM}$ expected for flight deck-based spacing. In order to maintain a reduced separation buffer not possible by non-FIM operations, a low frequency of FIM interruptions must be achieved. For the runway-based model, a reduction in the runway separation buffer would cause a corresponding, and undesirable, increase in the FIM interruption rate. Table 4 shows the runway separation buffers necessary to achieve a FIM interruption rate of $4.6 \%$. For the PHX arrival procedures and wind conditions, an average runway separation buffer of $0.25 \mathrm{NM}$ is necessary to achieve a FIM interruption rate of $4.6 \%$ across all arrival procedures and both landing directions. Like previous results, West Flow shows larger variability than East Flow. These results suggest that a reduction of the runway separation buffer will be less effective or even not possible if a low frequency of FIM interruptions is targeted.

Figure 8 illustrates a clear trade between the FIM interruption rate and runway throughput as a result of the multiple separation constraints and differing crossing speeds at those points. Using the meter fix-based paradigm reduces the FIM interruption rate prior to the meter fix, but it achieves a lower mean runway throughput than the other paradigms. Using the runway-based paradigm achieves better mean runway throughput, but causes FIM interruptions to occur earlier in the descent prior to the meter fix. The composite paradigm reflects the best overall behavior - it exhibits the highest mean runway throughput and avoids FIM interruptions by using the most restrictive scheduling constraint as the ASG. The composite paradigm exhibits a slightly higher $95 \%$ central range of runway throughput (reflecting the range of runway spacing due to different headwinds along final approach) than the runway-based paradigm, but its range is still modest. Therefore, it is recommended that the ASG be calculated using the most restrictive scheduling constraint along the FIM and Target aircrafts' shared arrival path. When the FIM and Target aircraft are assigned the same arrival procedure, this corresponds to using the larger of the meter fix and runway threshold desired spacing values.

\section{Table 4. Runway Separation Buffer for the Runway-Based Paradigm $(p<4.6 \%)$}

\begin{tabular}{|c|c|c|}
\cline { 2 - 3 } \multicolumn{1}{c|}{} & East Flow & West Flow \\
\hline EAGUL & $0.30 \mathrm{NM}$ & $0.44 \mathrm{NM}$ \\
\hline GEELA & $0.22 \mathrm{NM}$ & $0.00 \mathrm{NM}$ \\
\hline KOOLY & $0.24 \mathrm{NM}$ & $0.30 \mathrm{NM}$ \\
\hline MAIER & $0.29 \mathrm{NM}$ & $0.23 \mathrm{NM}$ \\
\hline
\end{tabular}

For FIM interruption rates in excess of $0.3 \%$, there are no significant inflection points in the curves show in Figure 8. These results are generally positive, because the FAA-reported arrival rates for PHX and other major airports in the NAS are approximately 36-48 aircraft per hour per runway during VMC periods and $10-20 \%$ lower during IMC periods [14]. The mean runway throughput values predicted for all three paradigms are within this range for the FIM interruption rates studied.

For the composite paradigm, the tradeoff is less pronounced. A reduction in the runway separation buffer increases the number of combinations of arrival procedure and wind condition that require the use of the meter fix spacing as the ASG instead of the runway spacing. As a result of this particular interaction, the PHX analysis predicted that a decreased runway separation buffer is only about $85 \%$ effective in increasing the mean runway throughput. Until multiple FIM clearances involving multiple Target aircraft, ABPs and ASGs can be communicated to the FIM aircraft or a dynamic 
model of the merging and spacing operations is incorporated into the FIM spacing algorithm, it is not likely that the runway separation buffer can be reduced, but requires more research to verify.

Using the composite paradigm may create a situation where the FIM and Target aircrafts' desired runway separation is no longer the same as the other non-FIM aircrafts'. By itself, this consequence is not an unacceptable behavior for arrival operations. Similar results occur whenever any separation constraint not associated with simple in-trail separation is applied. However, it is important that the controller's tools present a consistent view of the arrival plan. Both the schedule and its solution trajectories must reflect the scheduling constraints imposed by the FIM operation. The ASG should be a direct reflection of the predicted inter-arrival times at the ABP based upon the arrival schedule and its corresponding trajectories. In this manner, the ASG calculation paradigms are more appropriately considered to be FIM scheduling paradigms.

\section{Alternative Solutions}

This section proposes alternative solutions for conducting the FIM operations in a manner more consistent with the arrival schedule to avoid FIM interruptions. These solutions represent modifications to the broader ATD-1 Concept of Operations rather than the simpler recommendations for time-based metering.

The first alternative is to delay the FIM initiation until past the meter fix. The issuance of the FIM clearance could still be given prior to or shortly after top-of-descent in order to keep the controller and pilot workload manageable. All of the paradigms assumed the FIM operation would be initiated outside of the meter fix near top-of-descent and then terminated at the final approach fix. Limiting the FIM operation to the later portion of the arrival avoids the transition from $5 \mathrm{NM}$ en route separation to $3 \mathrm{NM}$ terminal separation. As a result, the runway-based paradigm would be able to avoid upstream FIM interruptions due to the desired meter fix separation. However, the differing spacing requirements at the meter fix and runway threshold will still cause the initial spacing error (spacing at FIM initiation versus ASG) to exhibit non-zero statistical biases. An assumption of previous airborne spacing research has been that the initial spacing errors are unbiased, so the effect of this behavior must be investigated. Overall, this approach is promising as a potentially more reliable method to avoid interruptions while restricting the FIM operation to a single ASG. This FIM operation would be a moderate change to both NASA's ATD-1 Concept of Operation as well as the FAA's Interval Management Concept of Operations [2][10].

A second alternative is to use consecutive FIM clearances to communicate the scheduling constraints as they each become active. For example, the meter fix spacing would be communicated near top-ofdescent; spacing at an intermediate terminal fix would be communicated as the aircraft passed the meter fix; and finally, the runway or final approach fix spacing would be communicated as the aircraft was handed-off from the feeder controller to the final controller. This modified FIM operation would ensure that all of the scheduling constraints could be satisfied by the FIM operation. However, it is not believed that this approach will be manageable in a high-density, voice-only environment. Since Data Communications is not expected to be available for communication of these more complex FIM clearances in the mid-term timeframe, it is not considered an immediately feasible option.

Finally, a third potential mitigation to the schedule-related FIM interruptions is to perform FIM operations only when the wind conditions at the critical meter points are conducive to continued FIM operations. For example, FIM operations could be suggested by the air traffic scheduling automation only when the runway threshold time constraint dominates the meter fix time constraint. The arrival scheduler would need to estimate the FIM interruption rate in a manner similar to the analysis discussed above. This alternative solution is effectively the same as accepting a larger FIM interruption rate since some potential FIM operations would simply not be attempted. Overall, the FIM operational benefit, in terms of procedure availability, would be reduced similarly in both cases. Inevitably, some FIM operations predicted to be interrupted would be successful. Further analysis is needed to determine whether unnecessary unavailability is more operationally acceptable than repeated FIM interruptions. 


\section{Future Work}

An analytical model of the dynamic behavior of the FIM and Target aircraft spacing needs to be coupled with this analytical model of the static behavior of the ATD-1 arrival scheduler. This more sophisticated model should be used to determine the most feasible ASG for each FIM pair by trading between the associated runway throughput and FIM interruption rates. The results presented in this paper are considered an optimistic estimate of the FIM interruption rate due to scheduling constraints when the FIM aircraft achieves the single ASG perfectly.

Also, the analysis described in this study should be applied to other airports in the NAS. Figure 4 and Figure 5 demonstrate airspace-dependent behavior is present. It is important to determine if airports that lay elsewhere, such as within the jet stream, are more or less affected by the winds.

\section{References}

[1] Joint Planning and Development Office, 30 Sep 2010, "Concept of Operations for the Next Generation Air Transportation System," Version 3.2.

[2] Baxley, B. T, W. C. Johnson, H. N. Swenson, J. E. Robinson, T. Prevot, T. J. Callantine, J. Scardina, and M. Greene, 2013, "Air Traffic Management Technology Demonstration-1 Concept of Operations (ATD-1 ConOps)," Version 2.0, NASA TM-2013218040.

[3] Abbott, T. S., 2013, "An Overview of a Trajectory-Based Solution for En Route and Terminal Area Self-Spacing: Fourth Revision," NASA CR-2013-218044.

[4] Barmore, B., T. Abbott, K. Krishnamurthy, 29 Aug-3 Sep 2004, "Airborne Managed Spacing in Multiple Arrival Streams," $24^{\text {th }}$ International Congress of Aeronautical Sciences (ICAS), Yokohama, Japan.

[5] Federal Aviation Administration, 22 Aug 2013, "Air Traffic Control," FAA JO 7110.65U Change 3, pp. 5-5-1 - 5-5-6.

[6] Kupfer, M., T. Callantine, L. Martin, J. Mercer, and E. Palmer, 14-17 Jun 2011, "Controller Support Tools for Schedule-Based Terminal-Area Operations," 9th USA/Europe Air Traffic
Management Research and Development Seminar (ATM2011), Berlin.

[7] Callantine, T. J., M. Kupfer, L. Martin, and T. Prevot, 10-13 Jun 2013, "Simulations of Continuous Descent Operations with Arrival-Management Automation and Mixed Flight Deck Interval Management Equipage," 10th USA/Europe Air Traffic Management Research and Development Seminar (ATM2013), Chicago.

[8] Thipphavong, J., J. Jung, H. Swenson, L. Martin, M. Lin, and J. Nguyen, 5-10 Oct 2013, "Evaluation of the Terminal Sequencing and Spacing System for Performance-Based Navigation Arrivals," 32nd Digital Avionics Systems Conference (DASC), Syracuse, pp. 1A2-1-1A2-16.

[9] Federal Aviation Administration, 1 Feb 2014, "Aviation System Performance Metrics (ASPM)," aspm.faa.gov/apm/sys.

[10] Federal Aviation Administration, 19 Dec 2012, "Arrival Interval Management - Spacing (IMS) Concept of Operations for the Mid-Term Timeframe," PMO-010, Revision 01.

[11] Zelinski, S., 14-18 Oct 2012, "Benefits of Precision Scheduling and Spacing for Arrival Operations," 31st Digital Avionics Systems Conference (DASC), Williamsburg, VA, pp. 3A5-13A5-15.

[12] Murdoch, J. L., S. R. Wilson, C. E. Hubbs, J. W. Smail, 19-22 Aug 2013, "Acceptability of Flight Deck-Based Interval Management Crew Procedures," AIAA Modeling and Simulation Technologies Conference (MST), Boston.

[13] Federal Aviation Administration Air Traffic Organization (ATO) En Route Services, August 2007, "Surveillance and Broadcast Services Benefits Basis of Estimate," FAA-2007-29305-0013-1.

[14] Federal Aviation Administration, 31 Jan 2014, “ATSCC OIS System,” www.fly.faa.gov/ois.

\section{3rd Digital Avionics Systems Conference} October 6-10, 2014 


\section{Submitting Your Final Paper for Review and Approval}

As soon as you have completed the final version of your paper, submit it electronically at:

www.dasconline.org. This must be done no later than August 19, 2014 to ensure adequate time for review and publication.

Name the file using your abstract number and up to the first five (5) letters of your last name (i.e., for abstract number 115, author Michael L. Johnson would save his file as: 115 johns.doc, where .doc is the default three letter extension applied by Microsoft ${ }^{\star}$ Word.) Omit any spaces in your last name when naming your file (i.e., for abstract number 122, author Tom de Jong would save his file as: 122dejon.doc).

If you cannot submit the paper via www.dasconline.org, or your paper is more than $25 \mathrm{MB}$, two additional options are available:

- Email (if less than $15 \mathrm{MB}$ in size); or

- Send a CD, or DVD by postal service to the Publications Editor at the address below, under heading "Additional Documentation Requirements"

Please indicate your use of Mac or PC, your full name, address, and phone number, "33rd DASC," and the file name of your paper.

The point of this conference is to foster a healthy information exchange among government and industry representatives. If you upload a paper for publication, then at least one of the authors listed on the paper is expected to attend and present that paper. IEEE reserves the right to exclude a paper from distribution after the conference (e.g., removal from IEEE Xplore) if the paper is not presented at the conference. Please register for the conference at www.dasconline.org.

\section{IEEE Copyright Form}

All DASC papers are published through IEEE. Download the 33rd DASC official IEEE Copyright Form from www.dasconline.org. Complete, and sign the form. Submit the form to the Publications Editor, via one of the following options:

- Scan to pdf or graphic file (jpg, gif, png, tif) and email

- Fax

- Postal service (Please note: Overnight delivery service is recommended; in case their tracking service is required for lost documents).

To:

Tony Rossetti

ALR International

3311 Dupree Avenue

Orlando, FL 32806-3411

pub.editor@dasconline.org

Fax: 888.338.3537 (toll free)

All required documents must be received no later than August 19, 2014 to ensure adequate time for review and publication. 
Please be aware of any additional release requirements that you may have above and beyond the ones imposed by this conference. Of particular interest are project sponsor and customer releases. Many companies also have their own publication release processes that must be satisfied prior to submitting the paper to the 33rd 